\title{
Effect of RSK4 on biological characteristics of colorectal cancer
}

\author{
Qingqing $\mathrm{Ye}^{1 \dagger}$, Xuan Wang ${ }^{2 \dagger}$, Min $\mathrm{Jin}^{3}$, Meng Wang ${ }^{2}$, Yan $\mathrm{Hu}^{2}$, Shihu $\mathrm{Yu}^{2}$, Yonghua Yang ${ }^{2}$, Jiyuan Yang ${ }^{2 *}$ \\ and Jun Cai ${ }^{2^{*}}$
}

\begin{abstract}
Background: This study aimed to investigate the expression of P90 Ribosomal Protein S6 kinase 4 (RSK4) in colorectal cancer cells and its biological function.

Methods: We selected early SW480 and HCT116 colorectal cancer cell lines, using Lipofectamine ${ }^{\text {TM }} 2000$ transfection reagent carrying RSK4 gene transfected into cells to establish the colorectal cancer cell lines with high expression of RSK4. RT-PCR and western blot (WB) analysis confirmed RSK4 expression in SW480 and HCT116 cancer cell lines. We used methylthiazoltetrazolium (MTT) assay and flow cytometry to detect the proliferation of colorectal cancer cells. After transfection of RSK4, the effect of RSK4 on the RNA levels associated with epithelial-mesenchymal transition (EMT) of colorectal cancer cells was analyzed by real-time fluorescence quantitative PCR and the expression of EMTrelated protein was detected by WB analysis.
\end{abstract}

Results: After transfection of RSK4 overexpression, the MTT assay detected that RSK4 could significantly inhibit the growth of colorectal cancer cells in vitro; flow cytometry detected that S-phase cells decreased significantly, and G0/1 cells increased significantly $(P<0.05)$. The invasion ability of SW480 and HCT116 cells transfected with RSK4 was markedly lower than that in the control group, and the difference was statistically significant $(P<0.05)$. Fluorescent quantitative PCR and WB analysis showed that the expression of EMT-associated molecular E-cadherin was remarkably increased and the expression of Snail was significantly decreased $(P<0.01)$.

Conclusion: RSK4 gene in colorectal cancer cell lines with low expression of RSK4 after transfection can inhibit the growth and invasion of tumor cells. RSK4 gene may inhibit EMT and inhibit metastasis of colorectal cancer cells, may be a potential tumor suppressor gene and inhibit tumor distant metastasis, and may provide the biological basis for new therapeutic targets.

Keywords: RSK4, Colorectal cancer, Tumor suppressor gene, Epithelial-mesenchymal transition, E-cadherin

\section{Background}

Colorectal cancer is the most common malignant tumor among gastrointestinal tract cancer. The distant metastasis of colorectal cancer cells is closely related to the morbidity and mortality of the disease. Further study of the pathogenesis of colorectal cancer is urgently being explored, to discover more effective methods for prevention, diagnosis, and treatment of the progression of cancer. RSK4 is a recent discovery gene located on X chromosome of linkage genes [1]. RSK is the downstream target

\footnotetext{
* Correspondence: jiyuanyangchina@163.com; caijun0540@163.com ${ }^{\dagger}$ Qingqing Ye and Xuan Wang contributed equally to this work.

${ }^{2}$ Department of Oncology, First Affiliated Hospital of Yangtze University, Jingzhou 434000, Hubei, China

Full list of author information is available at the end of the article
}

of Raf-MEK-ERK signaling pathways, which belongs to the serine threonine kinase family. So far, four RSK members have been found in this family, They were play an important role in various life activities such as regulating cell proliferation, movement, growth, survival, and invasion.

The literature has reported that RSK4 gene can inhibit different breast cancer cell line growth, invasion, apoptosis, and metastasis both in vitro and in vivo [2-7]. Although it may be a suppressor gene of cancer, there are opposite reports that exogenous RSK4 gene had no obvious inhibitory effect on breast cancer progress [8]. Dewdney et al reported RSK4 expression in normal endometrium, and that the expression is lower or missing in endometrial carcinoma [9]. RSK4 is highly methylated in endometrial 
carcinoma cell lines and endometrial carcinoma compared with normal endometrium. It is not certain currently whether RSK4 is a tumor suppressor gene for endometrial cancer. Fan et al. and another 16 studies have found that RSK4 plays an important role in the prognosis of renal cell carcinoma [2]. What role does RSK4 play in the end? Sun et al. [10] carried out a series of studies on the genes which can encode RSK4, suggesting that RSK4 is an oncogene or tumor suppressor gene depending on many factors. In our previous study, we have demonstrated that RSK4 expression was low in colorectal cancer, and immunohistochemical analysis found that the expression of RSK4 protein in colorectal cancer is closely related to the clinical pathology of lymph node metastasis, tumor grade, and clinical stage [11]. Therefore, we will have a deep discussion to clarify RSK4 gene play a role in the proliferation and invasion of colorectal cancer cells.

\section{Methods}

\section{Cell culture}

Human colorectal cancer cell lines SW480 and HCT116 are held by our unit. They were cultured in RPMI 1640 medium containing 10\% fetal bovine serum (FBS) and cultured in an incubator containing 5\% $\mathrm{CO}_{2}$ and $95 \%$ humidity. The culture medium was changed every 2 or 3 days depending on the state of cells, and the cells were passaged when they grew well and the density was between 80 and $90 \%$.

\section{Transient cell transfection plasmid}

Plasmid was extracted with a plasmid extraction kit without endotoxin. Transfection was carried out following the instructions of Lipofectamine ${ }^{\mathrm{Tw}} 2000$. A density of $5 \times 10^{5}$ cells was inoculated into the six-hole plate 1 day before transfection. The cells were transfected with $4 \mu \mathrm{g}$ plasmid and $10 \mu \mathrm{l}$ Lipofectamine ${ }^{\mathrm{Tw}} 2000$ reagent; the control group was not transfected, and the negative control group was transfected with empty plasmid. After 4-6 h, the intact medium containing FBS was changed to continue culture.

\section{RT-PCR}

The mRNA expression of RSK4 and EMT-related molecules in the SW480 and HCT116 cell lines respectively was detected by RT-PCR. Trizol was used to extract total cellular RNA, $3 \mu \mathrm{g}$ total RNA for each cell. cDNA first-strand synthesis was performed by reverse transcription according to the reverse transcription kit. Primers were designed using Primer 5 software according to the gene sequences reported by GenBank and the literature. Each cell was subjected to three independent repeated trials. Finally, we took the average value of the three experiments. The primer sequences are presented in Table 1.
Table 1 Sequence of primers including EMT-related molecules, RSK4, and $\beta$-actin

\begin{tabular}{ll}
\hline Molecule & Primer \\
\hline RSK4 forward & AATACTACCATTCGCTCCTCA \\
RSK4 reverse & TTACAGGCCAGTTGATGTCGC \\
H-actin forward & GTCCACCGCAAATGCTTCTA \\
H-actin reverse & TGCTGTCACCTTCACCGTC \\
H-CLDN-2 forward & GGCTTTCCACAGAGAGACGGG \\
H-CLDN-2 reverse & CAAGCAGCCTCAAGAAGGCAT \\
H-E-cadherin forward & CTITGACGCCGAGAGCTAC \\
H-E-cadherin reverse & TTGAATCGGGTGTCGAGGG \\
H-P53 forward & CCTCCTCAGCATCTTATCCG \\
H-P53 reverse & TTCCGTCCCAGTAGATTACCA \\
H-Snail forward & AGGACACATTAGAACTCACACGG \\
H-Snail reverse & CGAGTAAACATTGATTGCGTCAC \\
H-TGF- $\beta$ forward & TACCTGAACCCGTGTTGCTCT \\
H-TGF- $\beta$ reverse & TTCCCCTCCACGGCTCAAC \\
H-twist forward & TGGCCTGCAAAACCATAGTCA \\
H-twist reverse & AGACACCGGATCTATTTGCAT \\
H-Vimentin forward & TCAGAATATGAAGGAGGAAATGGC \\
H-Vimentin reverse & GAGTGGGTATCAACCAGAGGGAGT
\end{tabular}

EMT epithelial-mesenchymal transition, RSK4 P90 Ribosomal Protein 56 kinase 4 , TGF- $\beta$ transforming growth factor beta

\section{Western blot analysis}

Detection of the protein level of RSK4 and EMT-related molecules in colorectal cancer cell lines SW480 and HCT116 was by WB analysis. RIPA pyrolysis liquid was used to extract protein from the cells $72 \mathrm{~h}$ after transfection, following the operating procedures in accordance with the instructions for RIPA lysate. The BCA method was used for measuring the protein concentration. After electrophoresis, the protein was transferred to the PVDF membrane, with 5\% dried skimmed milk powder as a blocking reagent, mouse monoclonal antibody (1:2000), HRP- Goat Anti-Rabbit Secondary Antibody (1:4000). Before adding ECL luminous liquid and subsequent exposure in a floating bath.

\section{Methylthiazoltetrazolium assay}

MTT assay was used to detect the growth of cells before and after transfection. The exponential growth of cells (nontransfection group, blank plasmid group, and transfected group) was inoculated in the culture dish. After $12,24,48,72$, and $96 \mathrm{~h}$, the absorbance at $490 \mathrm{~nm}$ was measured by a microplate reader, and the killing rate of the cells was calculated.

\section{Flow cytometry to detect the cell cycle}

Trypsin was used to digest cells in logarithmic growth. A sample of $10^{6}$ cells was suspended in PBS in a single cell suspension liquid. Cells were then fixed with ethanol 
precooled to $-20{ }^{\circ} \mathrm{C}$, kept at $4{ }^{\circ} \mathrm{C}$ overnight, centrifuged at $2000 \mathrm{r} / \mathrm{min}$, and washed twice with PBS. Flow cytometry was used to analyze the cell cycle after adding propidium iodide (PI) staining solution and incubating for $30 \mathrm{~min}$ at $37^{\circ} \mathrm{C}$.

\section{Transwell invasion assay}

BD Matrigel was melted at $4{ }^{\circ} \mathrm{C}$, and the medium and transwell plate were precooled. Then $100 \mu \mathrm{l}$ diluted Matrigel was added into each upper transwell chamber, and incubated at $37^{\circ} \mathrm{C}$. The cells were collected and washed twice, and then suspended and counted, and the cells were diluted to $1 \times 10^{6} / \mathrm{ml}$. The remaining medium was discarded and remaining matrix gum was washed away with DMEM. Then $600 \mu \mathrm{l}$ medium containing 10\% FBS was added into the transwell chamber underneath. A $100 \mu \mathrm{l}$ dilution of cell suspension liquid was added into the upper chamber. We cultured cells for $36-48 \mathrm{~h}$ at $37{ }^{\circ} \mathrm{C}$ with $5 \%$ $\mathrm{CO}_{2}$, washed with PBS, fixed with methanol, and dyed with crystal violet, washing off excess dyeing solution with tap water. Dyeing with crystal violet, the cells were mounted and viewed under a camera.

\section{Statistical methods}

Student's $t$ test was used to examine the differences in cell activity, gene transcription level, or protein level expression in each group. Results were shown as mean \pm standard deviation (mean $+\mathrm{SEM}$ ). There was significant statistical difference when $P<0.05$.

\section{Results}

\section{Overexpression of RSK4 and detection of mRNA and} protein in colon cancer cells

Our previous studies have shown low expression of RSK4 protein in colorectal cancer patients. We constructed RSK4 overexpression plasmid and transfected RSK4 gene into colorectal cancer cell lines (SW480 and HCT116). After $48 \mathrm{~h}$, the transfection efficiency was verified by real-time PCR. The results showed that, compared with normal cells and the blank plasmid group, the expression level of RSK4 in the RSK4 transfected groups increased in magnitude and the difference was statistically significant (Fig. 1). WB analysis showed SW480 and HCT116 transfected with blank plasmid with shallow protein bands, suggesting low expression of RSK4 protein. The color of these bands became deep when transfected with overexpression plasmid, suggesting that protein expression increased after RSK4 transfection (Fig. 2).

\section{Overexpression of RSK4 could inhibit the growth of colorectal cancer cells}

In order to confirm whether RSK4 of colorectal cancer cells has a growth inhibiting effect in vitro, we transfected RSK4

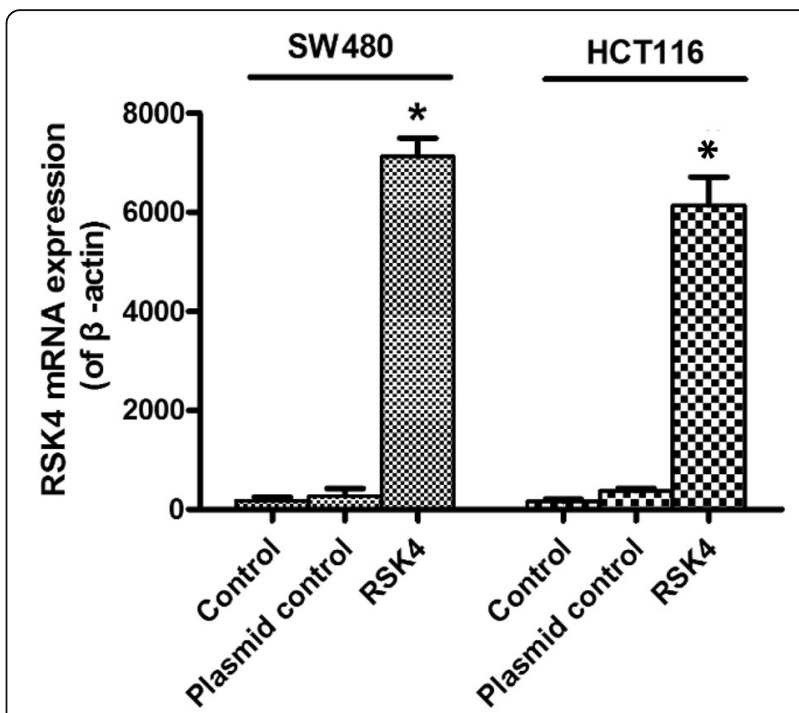

Fig. 1 Validation of transfection efficiency in SW480 and HCT116 cells. mRNA expression level in blank control group, empty plasmid group, and transfected RSK4 gene group detected by RT-PCR. Expression of RSK4 mRNA detected after $48 \mathrm{~h}(P<0.05)$. RSK4 P90 Ribosomal Protein 56 kinase 4

gene into SW480 cells (Fig. 3a) and HCT116 cells (Fig. 3b). The OD value was detected in the blank control group, empty plasmid group, and transfected RSK4 gene group by the MTT method at $12,24,36,48$, and $72 \mathrm{~h}$. The cell inhibition rate is equal to the blank group OD value minus that of the experimental group divided by the OD value of the blank group and multiplied by $100 \%$. The growth curve showed that overexpression of RSK4 can inhibit the growth of colorectal cancer cells in vitro, the inhibition at 48 and $60 \mathrm{~h}$ was about $60-70 \%$, while the normal group and blank plasmid group was about 10\%; the difference has statistical significance $(P<0.05)$ and the late suppression effect reached a plateau stage.

\section{Effects of RSK4 overexpression on the DNA synthesis cycle of colon cancer cells}

In order to further analyze the effect of RSK4 on the growth cycle of colorectal cancer cells, we used flow cytometry to analyze the cell cycle. The normal cells transfected with blank plasmid cells and transfected with RSK4 gene cells were cultured for $72 \mathrm{~h}$ and stained with propidium iodide (PI). We can detect the strength of DNA binding PI in cells by flow cytometry, which contributes to confirming the distribution of the cell cycle. The results showed that after transfection of RSK4, the percentage of S-phase cells among SW480 and HCT116 cells decreased, and the cells in the G0/1 phase increased obviously; the difference was statistically significant $\left({ }^{*} P<0.05\right)$ (Fig. 4a-d) (Table 2). 


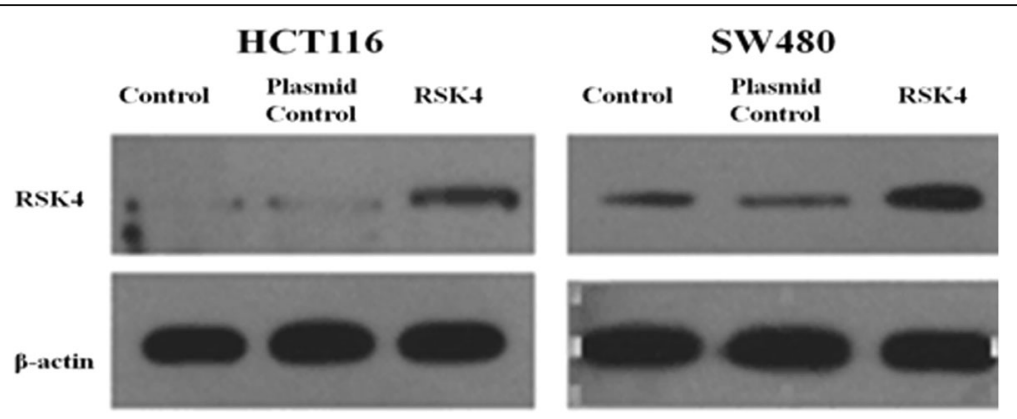

Fig. 2 Validation of RSK4 transfection efficiency in SW480 and HCT116 cells. Expression level in blank control group, empty plasmid group, and transfected RSK4 gene group detected by WB analysis. Expression of RSK4 protein detected after $48 \mathrm{~h}\left({ }^{*} P<0.05\right)$. RSK4 P90 Ribosomal Protein S6 kinase 4

\section{Effects of RSK4 overexpression on invasion and} metastasis of colorectal cancer cell

The discussed experiments revealed the effect of RSK4 on the growth and proliferation of colorectal cancer cells in various aspects. Therefore, can RSK4 affect the invasion ability of colorectal cancer cells in vitro? A transwell invasion assay showed that the invasion ability of transfection of RSK4 in SW480 cells and in HCT116 cells was significantly lower than in the control group; the difference was statistically significant (Fig. 5). These phenomena show that overexpression of RSK4 can reduce the invasiveness of colorectal cancer cells.

\section{The effect of RSK4 on the expression of target gene} mRNA

According to a large number of reports, RSK4 plays an important role in tumor invasion and metastasis, and tumor invasion and metastasis are closely related to epithelial-mesenchymal transition of tumor. Therefore, is the progress of RSK4 in tumor suppression associated with epithelial-mesenchymal transition? In order to determine whether RSK4 regulates these EMT-related genes, our first step was detection of the expression of mRNA (Claudin-2, E-cadherin, P53, Snail, TGF- $\beta$, Twist, and Vimentin) in the SW480 and HCT116 normal groups, empty plasmid group, and transfected RSK4 group by real-time quantitative PCR.

In the SW480 and HCT116 cell lines, we found high expression of E-cadherin in the transfected RSK4 group, especially in the HCT116 cell line at up to 14 times. The SW480 and HCT116 cell lines showed low expression of Snail, while Claudin-2, P53, TGF- $\beta$, Twist, and Vimentin mRNA expression levels did not change (Fig. 6a, b). These results suggest that RSK4 may play an important role in tumor invasion, metastasis, and epithelial-mesenchymal transition by regulating E-cadherin and Snail.

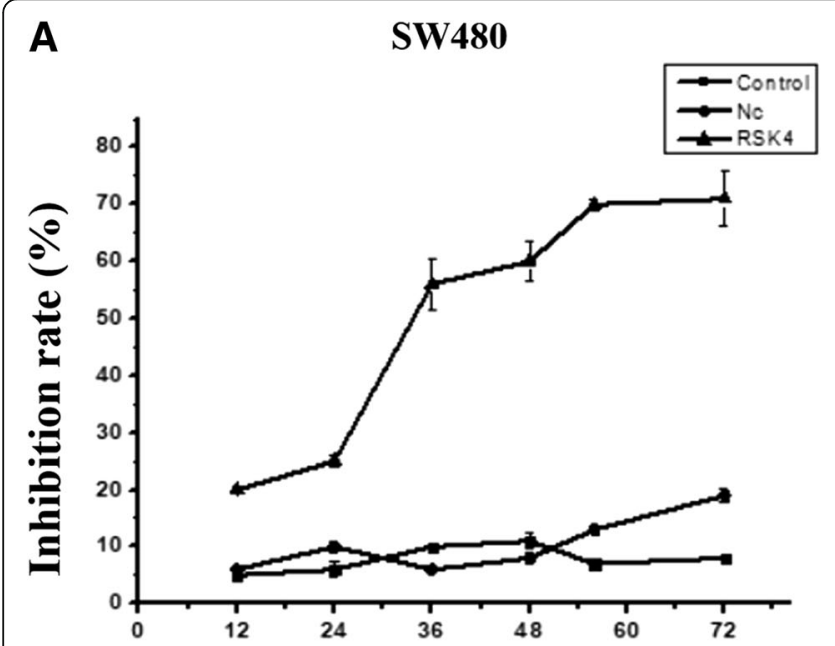

Incubation time (h)
B

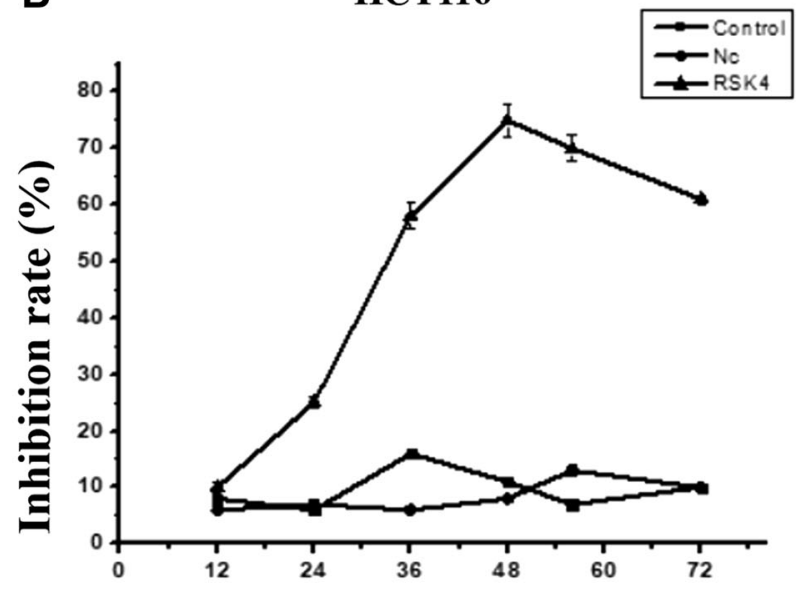

Incubation time (h)

Fig. 3 SW480 cell (a) and HCT116 cell (b) inhibition rate after transfection of RSK4. OD value detected at 12, 24, 36, 48, and 72 h. There was significant difference between control group and experimental group $\left.{ }^{*} P<0.05\right)$. RSK4 P90 Ribosomal Protein S6 kinase 4 


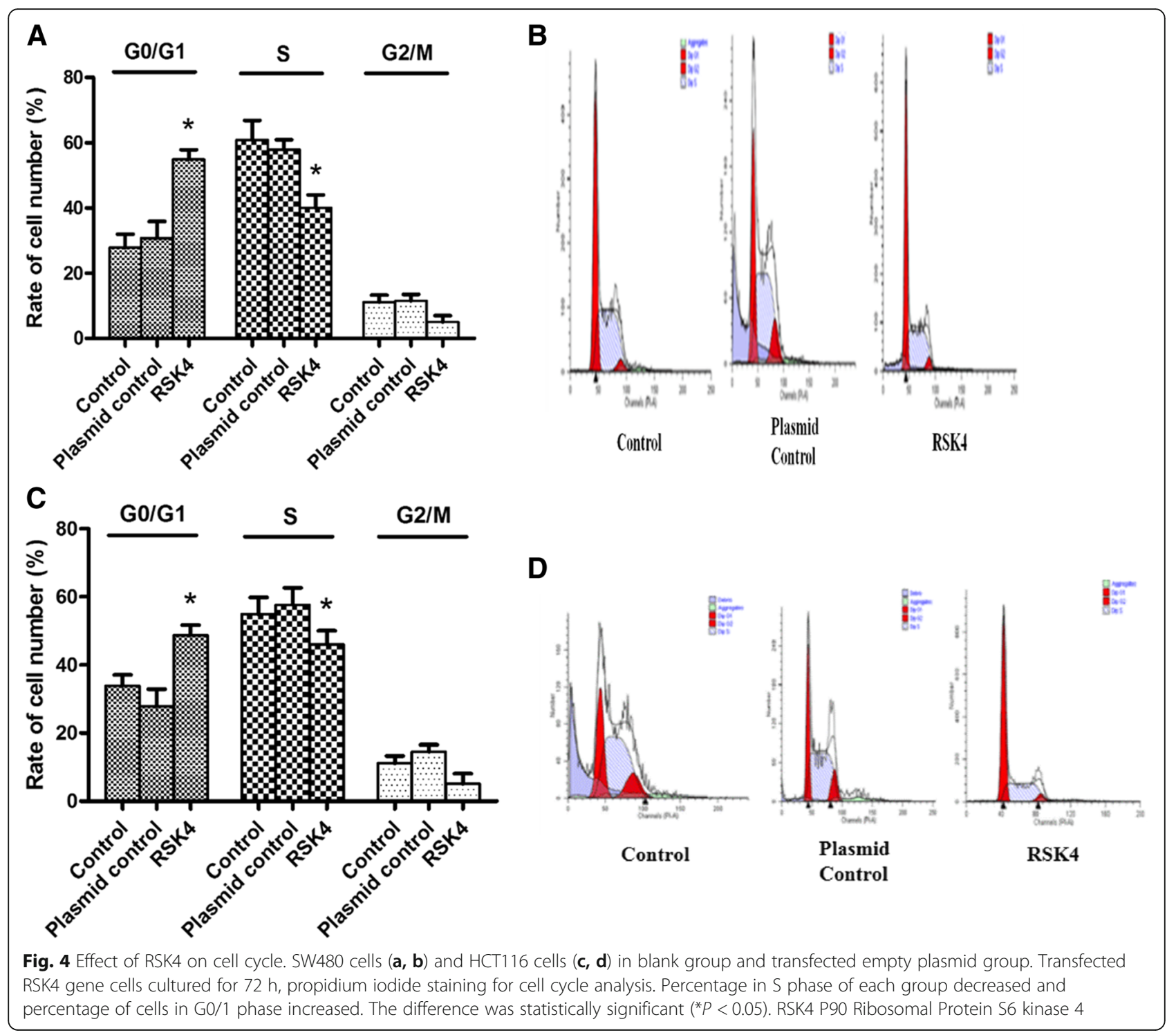

Table 2 Change of cell cycle after RSK4 transfection in SW480 and HCT16 cells

\begin{tabular}{llll}
\hline Group & G0/G1 phase & S phase & G2/M phase \\
\hline SW480 cell cycle (\%) & & & \\
$\quad$ Control & $27.91 \pm 4.1$ & $60.91 \pm 5.9$ & $11.17 \pm 2.1$ \\
Plasmid control & $30.82 \pm 5.1$ & $57.64 \pm 3.0$ & $11.54 \pm 2.0$ \\
RSK4 & $54.9 \pm 3.0$ & $40.05 \pm 4.0$ & $5.05 \pm 2.0$ \\
HCT 116 cell cycle (\%) & & & \\
Control & $33.91 \pm 3.2$ & $54.91 \pm 4.9$ & $11.17 \pm 2.1$ \\
Plasmid control & $27.82 \pm 5.1$ & $57.64 \pm 5.0$ & $14.54 \pm 2.0$ \\
RSK4 & $48.8 \pm 3.0$ & $46.1 \pm 4.0$ & $5.1 \pm 3.0$ \\
\hline
\end{tabular}

Change of cell cycle was detected $72 \mathrm{~h}$ after RSK4 transfection among different groups in SW480 and HCT16 cells ( $n=3$, mean \pm standard deviation) RSK4 P90 Ribosomal Protein S6 kinase 4
Effect of the expression level of RSK4 on the protein level We also examined the effects of RSK4 on the translation of genes (Claudin-2, E-cadherin, P53, Snail, TGF- $\beta$, Twist, and Vimentin) by western blot analysis. We again showed high expression of E-cadherin protein in the transfected RSK4 group, and low expression of Snail protein in the SW480 and HCT116 cell lines, while the protein levels of Claudin-2, P53, TGF- $\beta$, Twist, and Vimentin did not change (Fig. $7 \mathrm{a}-\mathrm{c}$ ). This result is consistent with the results of the PCR analysis.

\section{Discussion}

The incidence of colorectal cancer is increasing year by year in developed countries and developing countries. It is the third most common tumor in the world, and the mortality rate is fourth in the world [12]. In recent years, although the operation has made considerable progress, 


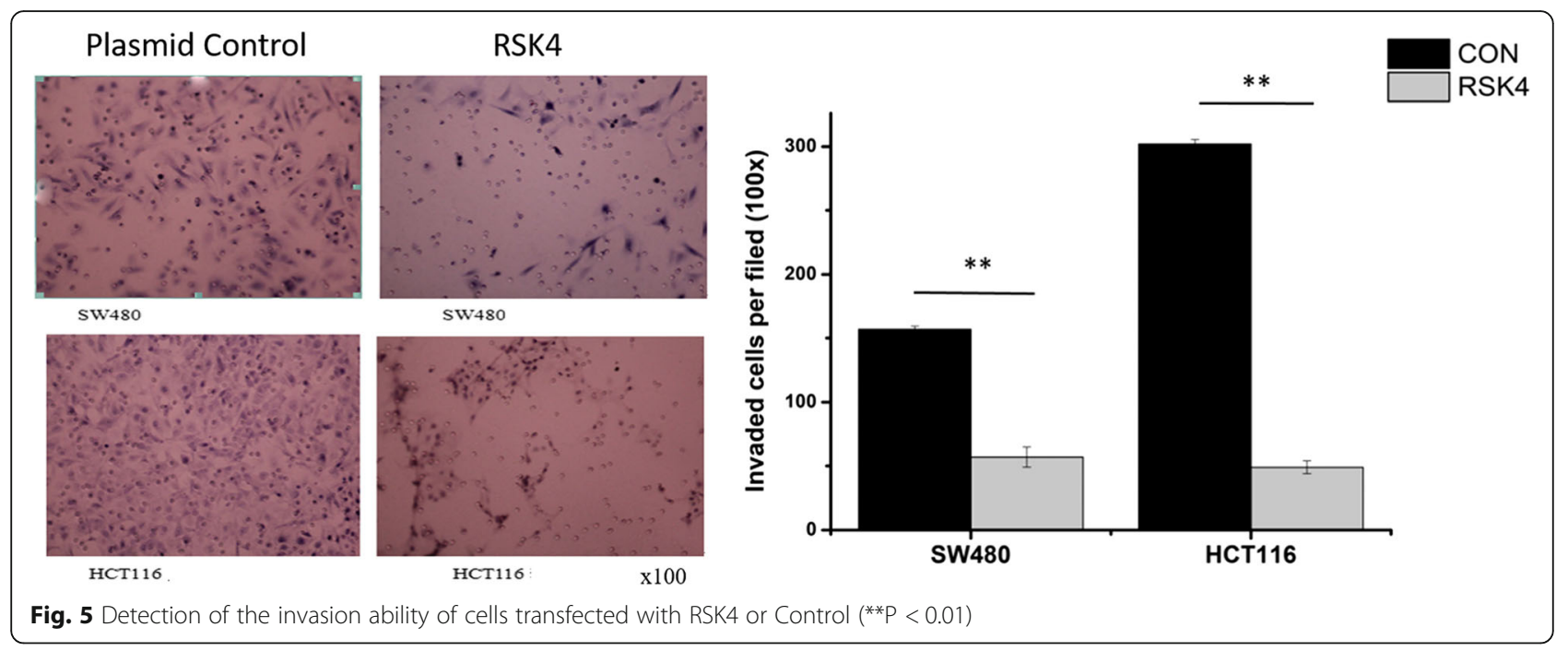

radiotherapy and chemotherapy need to be constantly updated. Various detection methods, biotherapy and targeted therapies for colorectal cancer are emerging, but without a revolutionary improvement, and they did not reach the expected results with a low 5-year survival rate. How to select specific molecular targets to attack tumors has become a difficult problem in current studies. Although bevacizumab and cetuximab achieved certain efficacy, they all encountered bottlenecks [13-17]. Finding a new target for colorectal cancer is imminent [18].

The Raf-MEK-ERK signaling pathway is a hot spot in colorectal cancer research. P90 ribosomal S6 Kinase (RSK) belongs to the serine kinase family, and is an important downstream target gene of the Raf-MEK-ERK signaling pathway. RSK4 is a linkage gene located on the X chromosome [19], and overexpression of RSK3 or RSK4 supports proliferation upon PI3K inhibition both in vitro and in vivo [20]. The $\mathrm{X}$ chromosome-linked oncogenes and tumor suppressor genes are regulated at the individual gene level. RSK4 inhibits the transcriptional activation of specific targets by effects on ERK, thereby inhibiting cell growth. Lopez-Vicente et al. [21] reported that expression of RSK4 decreased in colon cancer and renal cell carcinoma. At the subcellular level, RSK1-3 was found in the cytoplasm of quiescent cells, and a considerable part of the protein can be transferred to the nucleus if it receives stimulation. RSK4 mainly settles in the cytoplasm, and not in the nucleus from the stimulus of mitogen. The basic and clinical research of

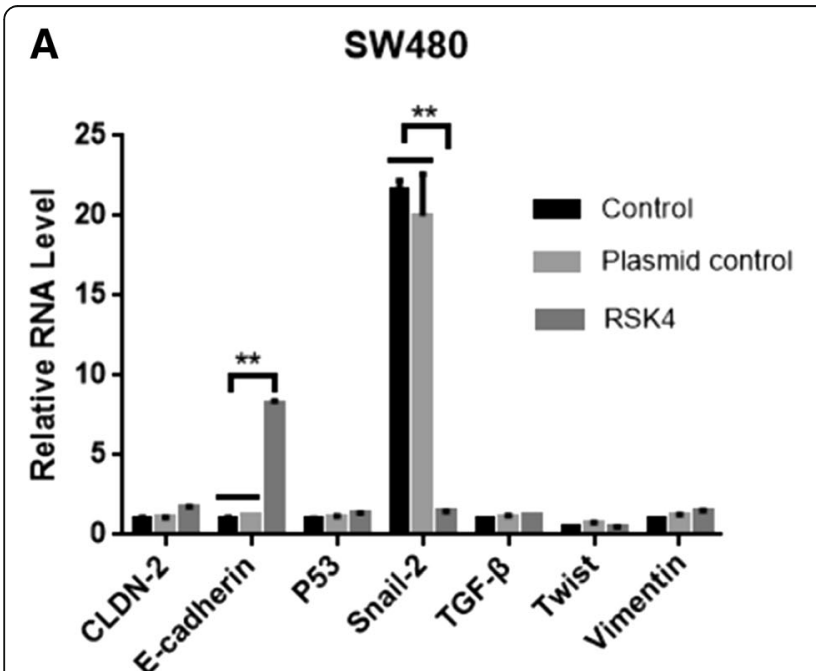

B

HCT116

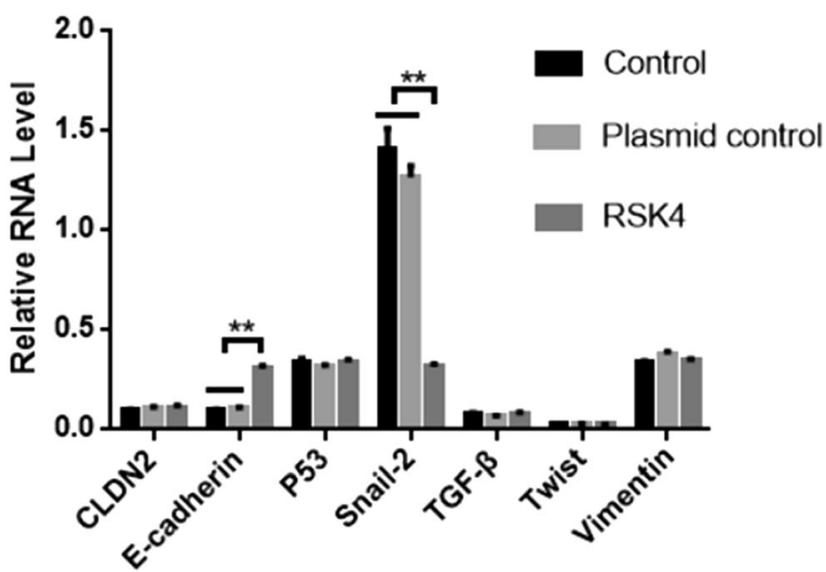

Fig. 6 Real-time quantitative PCR analysis of effect of RSK4 on EMT-related molecule mRNA in SW480 cells (a) and HCT116 cells (b). Expression of E-cadherin increased significantly, and expression of Snail decreased significantly ${ }^{* *} P<0.01$ ). RSK4 P90 Ribosomal Protein S6 kinase 4 , TGF- $\beta$ transforming growth factor beta 


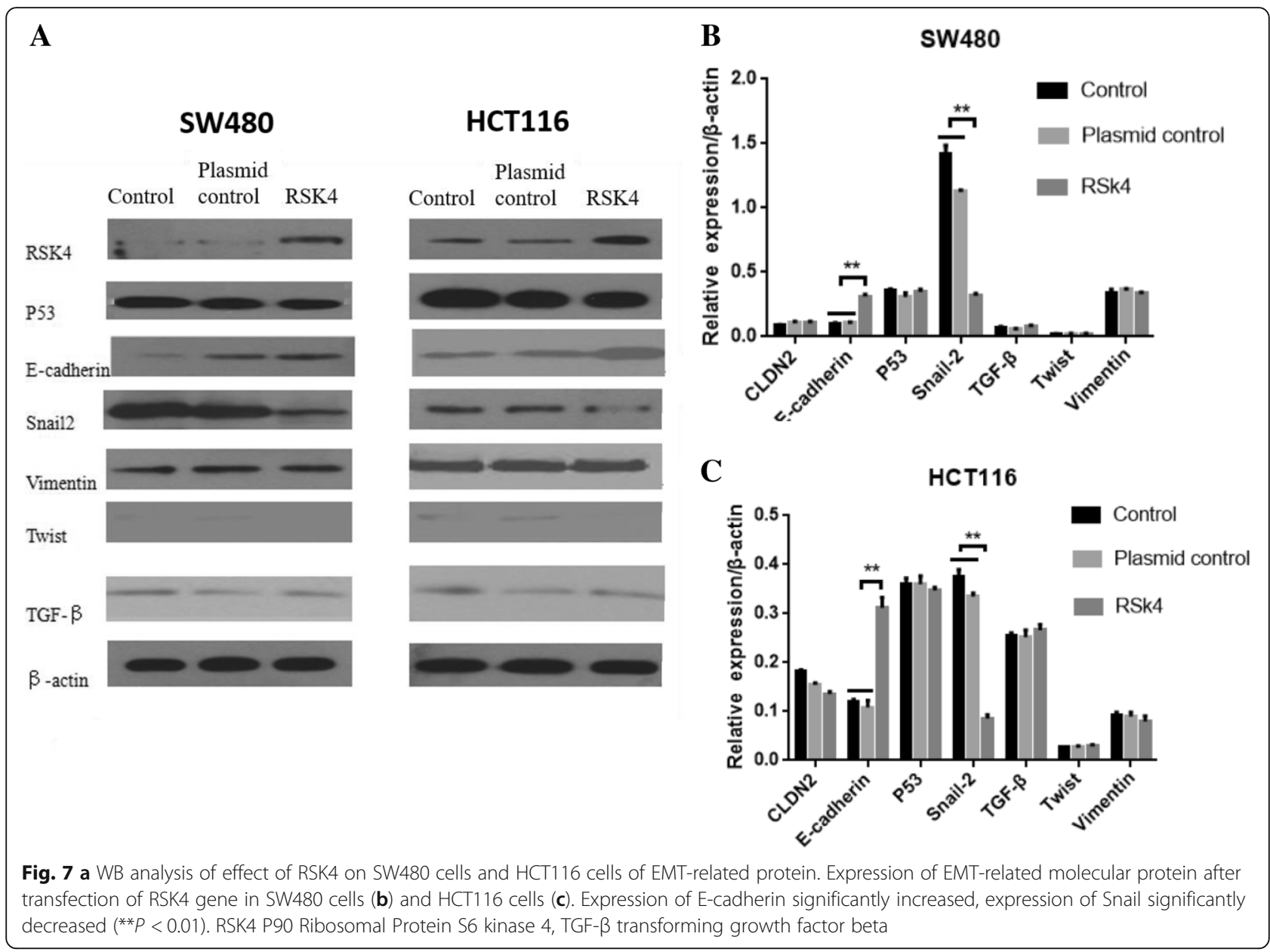

RSK4 in colorectal cancer is low, and only three papers have been reported at home and abroad.

Our previous studies have shown that RSK4 protein is highly expressed in colorectal cancer tissues, but low expressed in colorectal tissues. RSK4 may be one of the important biomarkers for the early diagnosis, prognosis, and treatment of colorectal cancer [22]. We used RT-PCR to detect the RSK4 expression level in SW480 and HCT116 cells, and the results showed that RSK4 expression was low or undetectable in these two cell lines. After optimizing the conditions, the plasmid was successfully transfected into the cells, and the RNA was extracted after culture for $48 \mathrm{~h}$ in a $37{ }^{\circ} \mathrm{C}$ incubator. We transfected RSK4 into different colorectal cancer cell lines, and then explored the specific biological function of RSK4 in colorectal cancer. In 48-60 h of transfection of RSK4 gene, the inhibition rate of SW480 cells was about $70 \%$, while for the normal cells and blank plasmid group it was about $10 \%$; the difference was statistically significant, while the late suppression effect reached a plateau. In HCT116 cells, the cells grew slowly after transfection of RSK4 gene, and the inhibition rate reached about $60 \%$ after $60 \mathrm{~h}$ of culture, while the normal cells and blank plasmid groups grew well, indicating that overexpression of RSK4 can inhibit the growth of colorectal cancer cells in vitro. For the sake of further analysis of the effect of RSK4 gene on the cell cycle of colorectal cancer, the cell cycle was detected by flow cytometry. The results showed the cell ratio of S-phase in normal cells and cells transfected with blank plasmid at around 57\%. After RSK4 gene transfection, SW480 cells and HCT116 cells in the S phase significantly decreased to $40 \%$. After transfection of RSK4 gene, G0/G1 phase cells increased significantly, and the difference was also statistically significant $(P<0.05)$. These results indicate that RSK4 gene inhibits tumor cell growth by inhibiting cell protein synthesis and may be a potential tumor suppressor gene.

Studies have shown that RSK4 also negatively regulates receptor tyrosine kinase (RTK) signaling during embryogenesis, inhibiting Ras-ERK signal transduction and cell proliferation [7, 23], so RSK4 with very low expression in the most rapid growth cells, upregulating intracellular expression, can inhibit cell growth, similar to our study. Thakur et al found that the expression of exogenous RSK4 had no effect on the growth of breast cancer cells 
[8]. But there are reports of the opposite; abnormal expression of some X-linked genes including RSK4 was found in breast cancer [23], indicating that abnormal RSK4 has a carcinogenic effect. Dewdney et al have reported RSK4 expression in the normal endometrium, and the expression is lower or missing in endometrial carcinoma, compared with normal endometrium [9]. It is not clear that RSK4 hypermethylation is an anti-oncogene in endometrial cancer. Sun et al performed a series of studies on the genes encoding RSK4, suggesting that RSK is an oncogene or tumor suppressor gene, depending on many factors [10].

\section{Conclusion}

RSK4 is an X-linked gene. Although its function is not fully understood, there is a large amount of data showing that RSK4 is highly expressed in cancer cells which can reduce cell proliferation and mitosis and promote apoptosis [20, 24]. RSK4 is involved in multiple signaling pathways in cancer development. Therefore, the application of RSK4 in cancer therapy may have potential breakthroughs and become a new therapeutic target worthy of further study.

\section{Abbreviations}

EMT: Epithelial-mesenchymal transition; FBS: Fetal bovine serum; MTT: Methylthiazoltetrazolium; PI: Propidium iodide; RSK4: P90 Ribosomal Protein S6 kinase 4; RTK: Receptor tyrosine kinase

\section{Authors' contributions}

YQQ carried out the main experiment and statistical analysis. WX carried out the main experiment and drafted the manuscript. JM provided some advice and carried out part of the experiment. WM carried out part of the experiment. HY provided technical guidance. YSH performed statistical analysis. YYH provided experimental guidance. YJY designed the study and provided technical guidance and financial contribution. CJ designed the study, analyzed the feasibility of the study, and provided financial contribution. All authors read and approved the final manuscript.

\section{Ethics approval and consent to participate}

Not applicable.

\section{Consent for publication}

Not applicable.

\section{Competing interests}

The authors declare that they have no competing interests.

\section{Publisher's Note}

Springer Nature remains neutral with regard to jurisdictional claims in published maps and institutional affiliations.

\section{Author details}

${ }^{1}$ Department of Breast Surgery, First Affiliated Hospital of Yangtze University, Jingzhou 434000, Hubei, China. ²Department of Oncology, First Affiliated Hospital of Yangtze University, Jingzhou 434000, Hubei, China. ${ }^{3}$ Cancer Center, Union Hospital, Tongji Medical College, Huazhong University of Science and Technology (HUST), Wuhan, Hubei, China.
Received: 26 April 2018 Accepted: 9 August 2018

Published online: 22 December 2018

\section{References}

1. Romeo Y, Zhang X, Roux PP. Regulation and function of the RSK family of protein kinases. Biochem J. 2012;441:553-69. https://doi.org/10.1042/ bj20110289.

2. Fan $L$, et al. Ribosomal $s 6$ protein kinase 4 : a prognostic factor for renal cell carcinoma. Br J Cancer. 2013;109:1137-46. https://doi.org/10.1038/bjc.2013.463.

3. Lara $R$, et al. An siRNA screen identifies RSK1 as a key modulator of lung cancer metastasis. Oncogene. 2011;30:3513-21. https://doi.org/10.1038/onc. 2011.61.

4. Vesely P, Rovensky Yu A, Slavnaya IL. Cinemicrographic analysis of migration of normal and neoplastic rat cells seeded upon the grooved surface. Folia Biol. 1981;27:81-90.

5. Yang HW, et al. Effects of breast cancer cells stably overexpressing RSK4 on growth of transplanted human breast cancer in severe combined immunodeficiency mice. Zhonghua Yi Xue Za Zhi. 2012;92:1845-8.

6. Zhu J, et al. RSK4 knockdown promotes proliferation, migration and metastasis of human breast adenocarcinoma cells. Oncol Rep. 2015;34: 3156-62. https://doi.org/10.3892/or.2015.4291.

7. Jiang $Y$, et al. Aberrant expression of RSK4 in breast cancer and its role in the regulation of tumorigenicity. Int J Mol Med. 2017;40:883-90. https://doi. org/10.3892/ijmm.2017.3069.

8. Thakur A, et al. Aberrant expression of X-linked genes RbAp46, Rsk4, and Cldn2 in breast cancer. Mol Cancer Res. 2007:5:171-81. https://doi.org/10. 1158/1541-7786.mcr-06-0071.

9. Dewdney SB, et al. Aberrant methylation of the X-linked ribosomal S6 kinase RPS6KA6 (RSK4) in endometrial cancers. Clin Cancer Res. 2011;17: 2120-9. https://doi.org/10.1158/1078-0432.ccr-10-2668.

10. Sun Y, et al. Basic anatomy and tumor biology of the RPS6KA6 gene that encodes the p90 ribosomal S6 kinase-4. Oncogene. 2013;32:1794-810. https://doi.org/10.1038/onc.2012.200.

11. Cai J, et al. Low expression of RSK4 predicts poor prognosis in patients with colorectal cancer. Int J Clin Exp Pathol. 2014;7:4959-70.

12. Chen W, et al. Cancer statistics in China, 2015. CA Cancer J Clin. 2016;66: 115-32. https://doi.org/10.3322/caac.21338.

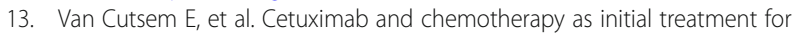
metastatic colorectal cancer. N Engl J Med. 2009;360:1408-17. https://doi. org/10.1056/NEJMoa0805019.

14. Yang JC, et al. A randomized trial of bevacizumab, an anti-vascular endothelial growth factor antibody, for metastatic renal cancer. N Engl J Med. 2003;349:427-34. https://doi.org/10.1056/NEJMoa021491.

15. Mishima $\mathrm{H}$, et al. FOLFIRI plus bevacizumab $5 \mathrm{mg} / \mathrm{kg}$ versus $10 \mathrm{mg} / \mathrm{kg}$ as second-line therapy in patients with metastatic colorectal cancer who have failed first-line bevacizumab plus oxaliplatin-based therapy: a randomized phase III study (EAGLE study). Jpn J Clin Oncol. 2012;42:134-8. https://doi. org/10.1093/jijco/hyr 180.

16. Petrelli $F$, et al. FOLFIRI-bevacizumab as first-line chemotherapy in 3500 patients with advanced colorectal cancer: a pooled analysis of 29 published trials. Clin Colorectal Cancer. 2013;12:145-51. https://doi.org/10.1016/j.clcc. 2013.04.006.

17. Heinemann $V$, et al. FOLFIRI plus cetuximab versus FOLFIRI plus bevacizumab as first-line treatment for patients with metastatic colorectal cancer (FIRE-3): a randomised, open-label, phase 3 trial. Lancet Oncol. 2014; 15:1065-75. https://doi.org/10.1016/s1470-2045(14)70330-4.

18. Sadanandam A, et al. A colorectal cancer classification system that associates cellular phenotype and responses to therapy. Nat Med. 2013;19: 619-25. https://doi.org/10.1038/nm.3175.

19. Dummler BA, et al. Functional characterization of human RSK4, a new 90-kDa ribosomal 56 kinase, reveals constitutive activation in most cell types. J Biol Chem. 2005;280:13304-14. https://doi.org/10.1074/jbc. M408194200

20. Serra $V$, et al. RSK3/4 mediate resistance to PI3K pathway inhibitors in breast cancer. J Clin Invest. 2013;123:2551-63. https://doi.org/10.1172/jci66343.

21. Lopez-Vicente $L$, et al. Regulation of replicative and stress-induced senescence by RSK4, which is down-regulated in human tumors. Clin Cancer Res. 2009;15:4546-53. https://doi.org/10.1158/1078-0432.ccr-08-3159.

22. Cai J, et al. Down-regulation of long noncoding RNA RP11-713B9.1 contributes to the cell viability in nonsmall cell lung cancer (NSCLC). Mol Med Rep. 2017;16:3694-700. https://doi.org/10.3892/mmr.2017.7026. 
23. Rafiee $\mathrm{M}$, et al. Down-regulation of ribosomal $\$ 6$ kinase RPS6KA6 in acute myeloid leukemia patients. Cell J. 2016;18:159-64.

24. Niskakoski A, et al. Epigenetic analysis of sporadic and lynchassociated ovarian cancers reveals histology-specific patterns of DNA methylation. Epigenetics. 2014;9:1577-87. https://doi.org/10.4161/ 15592294.2014.983374.

Ready to submit your research? Choose BMC and benefit from:

- fast, convenient online submission

- thorough peer review by experienced researchers in your field

- rapid publication on acceptance

- support for research data, including large and complex data types

- gold Open Access which fosters wider collaboration and increased citations

- maximum visibility for your research: over $100 \mathrm{M}$ website views per year

At $B M C$, research is always in progress.

Learn more biomedcentral.com/submissions 